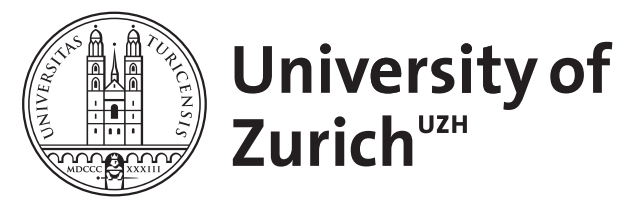

Zurich Open Repository and Archive

University of Zurich

University Library

Strickhofstrasse 39

CH-8057 Zurich

www.zora.uzh.ch

Year: 2006

Persische Reichsautorisation und Tora

Schmid, Konrad

Posted at the Zurich Open Repository and Archive, University of Zurich ZORA URL: https://doi.org/10.5167/uzh-68181

Journal Article

Originally published at:

Schmid, Konrad (2006). Persische Reichsautorisation und Tora. Theologische Rundschau, 71:494-506. 


\title{
Persische Reichsautorisation und Tora
}

\author{
Konrad Schmid (Zürich)
}

James W. Watts (Hg.), Persia and Torah. The Theory of Imperial Authorization of the Pentateuch, SBL.SS 17, Atlanta 2001.

\section{Das Problem}

Wie ist der Pentateuch zur Tora geworden? Dass der biblische Erzählzusammenhang von der Schöpfung der Welt (Gen 1) bis zum Tod Moses (Dtn 34) im Ostjordanland als eine Größe für sich zu gelten hat, ist ein faktisches Resultat der Kanonsgeschichte. Ebenso deutlich steht aber der Befund vor Augen, dass die kanonsgeschichtliche Ausgrenzung der Tora ursprünglich Zusammenhängendes - nämlich den über sie hinausweisenden Erzählfaden - durchtrennt hat. Ob man im literarhistorischen Vorfeld der Tora mit einem Hexateuch Gen-Jos oder einem Großgeschichtswerk Gen-2Kön rechnen darf oder muss, ist umstritten. Buchtechnisch sind solch umfangreiche Werke durchaus vorstellbar - sei es, dass man davon ausgeht, dass der entsprechende Text auf einer Rolle geschrieben war $^{1}$, oder dass man von einer entsprechen bibliothekarischen Ordnung von Teilrollen ausgeht ${ }^{2}$. Jedenfalls ist deutlich: Am Ende des Dtn steht Israel noch im Ostjordanland, an der Schwelle zum Gelobten Land. Das ist kaum der von vornherein sachlich intendierte Zielpunkt einer Großerzählung, die mit der Weltschöpfung begonnen hat. Der Dtn-Schluss markiert vielmehr eine sekundäre literarische Zäsur, zumal der Beginn des Josuabuches die zuvor abgebrochene Erzählung voraussetzt und zwanglos fortführt. Es stellt sich also die Frage nach den Gründen, Motiven und Triebkräften, die zur Formierung der Tora geführt haben, denn selbstverständlich ist dieser Vorgang offenkundig nicht. Man kann ihn mit einer gewissen Zuverlässigkeit in die Perserzeit datieren, denn deutliche literarische Reflexe auf den Zusammenbruch des Perserreichs wie in den Weltgerichtstexten des Corpus

\footnotetext{
1 Vgl. die Belege für ähnlich umfangreiche antike Buchrollen bei K. Schmid, Buchgestalten des Jeremiabuches. Untersuchungen zur Redaktions- und Rezeptionsgeschichte von Jer 30-33 im Kontext des Buches, WMANT 72, Neukirchen-Vluyn 1996, 38f Anm. 184-191 (Lit.).

${ }^{2}$ N.M. Sarna, The Order of the Books, in: C. Berlin (Hg.), Studies in Jewish Bibliography, FS I.E. Kiev, New York 1971, 407-413; ders., Ancient Libraries and the Ordering of the Biblical Books. A Lecture Presented at the Library of Congress, March 6, 1989, The Center for the Book Viewpoint Series 25, Washington 1989.
} 
propheticum $^{3}$ lassen sich nicht erkennen. Am Pentateuch ist zwar noch partiell bis in die Makkabäerzeit gearbeitet worden, allerdings kaum mehr durch Einfügung ganzer Erzählblöcke, sondern vielmehr durch einzelne Retuschen ${ }^{4}$.

Seit den achtziger Jahren des letzten Jahrhunderts ist ein theoretischer Zugang zu diesem Problem beliebt geworden, der Erkenntnisse des Zürcher Althistorikers Peter Frei $^{5}$ und - unabhängig davon entstanden - des jetzigen Tübinger Alttestamentlers Erhard Blum $^{6}$ aufgreift, und der mit dem Vorgang einer »persischen Reichsautorisation« des jüdischen Gesetzes rechnet. Der Sache nach ist diese Auffassung allerdings schon von einer Vielzahl anderer, früherer Forscher vertreten worden ${ }^{7}$. Dabei wird davon ausgegangen, dass die Formierung der Tora von außen, nämlich von der persischen Reichspolitik, angestoßen worden sei. Das Perserreich kannte - wiewohl bisweilen gesucht $^{8}$, vermutlich aber vergeblich ${ }^{9}$ - kein übergeordnetes Reichsrecht, sondern ein funktionales Äquivalent dieses Reichsrechts existierte wahrscheinlich lediglich in lokalen Gesetzen, die aber von der persischen Verwaltung autorisiert und so in den Status von Reichsrecht erhoben wurden. Für das perserzeitliche Juda wäre dann damit zu rechnen, dass eben die Tora (und gegebenenfalls bereits literarische Vorstufen von ihr) in diese Funktion einrückte.

Die aufgrund des früheren Publikationsdatums vor allem mit dem Namen Freis verknüpfte Theorie der »persischen Reichsautorisation« ist $\mathrm{zu}$ den erfolgreichsten Hypothesen in der alttestamentlichen Wissenschaft der letzten zwanzig Jahre zu zählen. Sie hat - neben zahlreichen Aufnahmen in Einzelstudien - auch Eingang in Einleitungen und Lehrbücher gefunden ${ }^{10}$. Nachdem sie sich zunächst großer und

\footnotetext{
${ }^{3}$ Vgl. dazu O.H. Steck, Der Abschluß der Prophetie im Alten Testament. Ein Versuch zur Frage der Vorgeschichte des Kanons, BThSt 17, Neukirchen-Vluyn 1991, 27f.37.83.

${ }^{4}$ Vgl. die Hinweise bei K. Schmid, Erzväter und Exodus. Untersuchungen zur doppelten Begründung der Ursprünge Israels in den Geschichtsbüchern des Alten Testaments, WMANT 81, Neukirchen-Vluyn 1999, 21 f mit Anm. 125 (Lit.).

${ }^{5}$ P. Frei, Zentralgewalt und Lokalautonomie im Achämenidenreich, in: ders./ K. Koch, Reichsidee und Reichsorganisation im Perserreich, OBO 55, Fribourg/Göttingen (1984) ${ }^{2} 1996,5-131$; ders., Die persische Reichsautorisation. Ein Überblick, ZAR 1 (1995), 1-35 (engl.: Persian Imperial Authorization: A Summary, in: J.W. Watts [Hg.], Persia and Torah. The Theory of Imperial Authorization of the Pentateuch, SBL.SS 17, Atlanta 2001, 5-40).

${ }^{6}$ Studien zur Komposition des Pentateuch, BZAW 189, Berlin/New York 1990, 333-360, vgl. die forschungsgeschichtliche Notiz 345 Anm. 42.; ders., E. Blum, Esra, die Mosetora und die persische Politik, in: R.G. Kratz (Hg.), Religion und Religionskontakte im Zeitalter der Achämeniden, VWGTh 22, Gütersloh 2001, 231-255, 250 Anm. 80.

${ }^{7}$ Vgl. die Hinweise bei Blum, Pentateuch, 346f Anm. 44.52; ders., Esra, 250 Anm. 78; U. Rüterswörden, Die persische Reichsautorsation der Thora: fact or fiction?, ZAR 1 (1995), 47-61, 51 Anm. 17-20, auf E. Meyer, H.H. Schaeder, M. Noth, E. Bresciani, U. Kellermann, W.Th. In der Smitten, u.a.

${ }^{8}$ Vgl. besonders die Theorie von A.T. Olmstead, A History of the Persian Empire, Chicago 1948, 119134; ders., Darius As Lawgiver, AJSL 51 (1934/1935), 247-249.

${ }^{9} \mathrm{Vgl}$. R. Frye, The History of Ancient Iran, HdO III/7, München 1983, 119.

${ }^{10}$ Vgl. z.B. R. Albertz, Religionsgeschichte Israels in alttestamentlicher Zeit, GAT 8/1.2, Göttingen 1992, 497-504; E.A. Knauf, Die Umwelt des Alten Testaments, NSK.AT 29, Stuttgart 1994, 171-175; E.
} 
zustimmender Rezeption erfreute, hat sich ihre Einschätzung, wie es scheint, in der jüngsten Vergangenheit verändert. Namentlich die kritische Diskussion in ZAR 1 (1995), in Einzelbeiträgen von E. Otto ${ }^{11}$ und H.C. Schmitt ${ }^{12}$ sowie nun in dem von J.W. Watts herausgegebenen Sammelband »Persia and Torah« hat einige Probleme aufgezeigt, die nähere Betrachtung verdienen. Besonders entschieden stellt E. Otto in seiner Besprechung des Sammelbandes von Watts fest ${ }^{13}$, dass $\gg$ die These .. durch die Fachiranisten einhellig abgelehnt worden« sei. Die Rezension mündet in die Feststellung: »Damit ist nun auch in der Alttestamentlichen Wissenschaft das Urteil, das die Iranistik längst gefällt hat, gesprochen. Der Pentateuch, das ist die Konsequenz, ist nicht Ergebnis persischer >Geburtshilfe<, sondern jüdischer Schriftgelehrsamkeit in persischer Zeit « (413). Dieses Urteil steht nicht für sich allein ${ }^{14}$, gleichwohl dürften die Dinge weniger klar liegen, als hier nahegelegt wird. Namentlich der iranistische Hauptgewährsmann der Kritiker Freis, J. Wiesehöfer ${ }^{15}$, ist - wie nachfolgend zu erläutern sein wird - in der Sache weniger weit von Frei entfernt, als er selbst und seine Rezipienten meinen.

Bei der Diskussion des Theoriekomplexes um die »persische Reichsautorisation« der Tora ist vorerst eine grundlegende Unterscheidung $\mathrm{zu}$ treffen. In ihm sind zwei Problemkreise verschränkt, die aber auseinanderzuhalten und mit Vorteil getrennt zu besprechen sind: Zum einen wird die Frage diskutiert, ob es überhaupt eine entsprechende Rechtsinstitution im Perserreich gegeben hat, und zum anderen steht zur

Zenger, Einleitung in das Alte Testament, KSTh 1,1, Stuttgart 1995, 39-42 (vgl. jetzt aber die Veränderungen in $\left.{ }^{5} 2004,129-131\right)$.

11 E. Otto, Kritik der Pentateuchkomposition, ThR 60 (1995), 163-191; 169 Anm. 5; ders., Die nachpriesterschriftliche Pentateuchredaktion im Buch Exodus, in: M. Vervenne (Hg.), Studies in the Book of Exodus. Redaction - Reception - Interpretation, BEThL 126, Leuven 1996, 61-111, 66-70; ders., Gesetzesfortschreibung und Pentateuchredaktion, ZAW 107 (1995), 373-392, 375 mit Anm. 14; ders., Die Tora des Mose. Die Geschichte der literarischen Vermittlung von Recht, Religion und Politik durch die Mosegestalt, Berichte aus den Sitzungen der Joachim Jungius-Gesellschaft der Wissenschaften e.V., Hamburg 2001, 51f.

${ }^{12}$ H.-C. Schmitt, Die Suche nach der Identität des Jahweglaubens im nachexilischen Israel. Bemerkungen zur theologischen Intention der Endredaktion des Pentateuch, in: J. Mehlhausen (Hg.), Pluralismus und Identität, VWGTh 8, Gütersloh 1995, 259-278, 263-267; ders., Das spätdeuteronomistische Geschichtswerk Gen I-2Regum XXV und seine theologische Intention, in: J.A. Emerton (Hg.), Congress Volume Cambridge 1995, VT.S 66, Leiden u.a. 1997, 261-279.

${ }^{13}$ Rez. J.W. Watts (Hg.), Persia and Torah, ZAR 8 (2002), 411-414 mit Verweis bes. auf J. Wiesehöfer (>Reichsgesetz< oder >Einzelfallgerechtigkeit $<$ ? Bemerkungen $\mathrm{zu}$ P. Freis These von der achaimenidischen >Reichsautorisation $<$ ZAR 1 [1995], 36-45).

${ }^{14}$ Vgl. z.B. C. Dohmen/M. Oeming, Biblischer Kanon warum und wozu? Eine Kanontheologie, QD 137, Freiburg u.a. 1992, 91 mit Anm. 3; N. Lohfink, Gab es eine deuteronomistische Bewegung?, in: W. Groß (Hg.), Jeremia und die »deuteronomistische Bewegung«, BBB 98, Weinheim 1995, 313-382 (= ders., Studien zum Deuteronomium und zur deuteronomistischen Literatur III, SBAB 20, Stuttgart 1995, 65142), 369f; J. Pakkala, Ezra the Scribe. The Development of Ezra 7-10 and Nehemia 8, BZAW 347, Berlin / New York 2004, 38.

${ }^{15}$ Wiesehöfer, ZAR 1995. 
Debatte, ob - gegebenenfalls - der Abschluss der Tora (bzw. die Formierung relevanter Vorstufen) damit zu erklären sei.

Beide Fragestellungen sind noch weiter zu differenzieren: So mutet zunächst die in der neuesten Forschungsdiskussion oft wahrnehmbare, bloße Alternative pro oder contra »Reichsautorisation« einigermaßen reduktionistisch an. In aller Regel wird dort, wo man sich gegen diese These ausspricht, tatsächlich nicht mehr als eine spezifische Fassung von ihr abgewiesen (s.u. II.). Dass es Vorgänge der Autorisierung lokaler Gesetze durch übergeordnete Instanzen im Perserreich, etwa durch den Satrapen, gegeben hat, lässt sich nicht bestreiten - so jedenfalls die wohl unumgängliche Minimalinterpretation der Trilingue aus Xanthos ${ }^{16}$, an der Frei seine These entwickelt hat: Auf der Stirnseite der lykisch und griechisch beschriebenen Stele veröffentlicht der Satrap Pixodaros den Beschluss der Gemeinde Xanthos, einen Kult für zwei karische Götter einzurichten, als seine eigene Anordnung auf aramäisch, der Reichssprache. Damit ist deutlich: Hier wird lokales Recht durch persische Übernahme seitens des Satrapen in den Status von Reichsrecht erhoben. Solche Vorgänge sind im übrigen innerhalb des Perserreiches namentlich in den kulturell weit entwickelten Gebieten wie Griechenland, Kleinasien, Juda oder Ägypten nur zu erwarten: Ein antikes Großreich wie dasjenige der Perser ist gar nicht ohne entscheidene Elemente von lokaler Autonomie zu verwalten, die aber durch die Zentralregierung gesteuert werden. Der Verwaltungsaufwand für die Einführung und Durchsetzung eines zentralen Reichsrechts wäre gar nicht zu leisten und ein entsprechender Versuch würde wohl überhaupt über sehr unsichere Aussichten verfügen.

Die Frage für den ersten Problemkreis kann also nicht lauten: Gab es eine »persische Reichsautorisation«?, sondern: Wie sind solche Vorgänge der Gewährung lokaler Autonomie von seiten der Perser, die zum einen nur zu erwarten und zum anderen unzweifelhaft belegt sind, adäquat zu beschreiben?

Entsprechend ist auch der zweite Problemkreis des Zusammenhangs zwischen der Formierung der Tora und der persischen Politik zu differenzieren: Auch hier kann es nicht darum gehen, diesen Zusammenhang pauschal abzuweisen oder zu befürworten, sondern es ist wiederum genau zu beschreiben, wie die Tora mit ihrem perserzeitlichen historischen Umfeld vernetzt ist und welche politischen Kräfte auf sie eingewirkt haben. Namentlich die von Otto propagierte Alternative »persischer >Geburtshilfe«« versus »jüdische Schriftgelehrsamkeit in persischer Zeit« dividiert zwei Faktoren auseinander, die einander nicht im Sinne eines »entweder-oder«, sondern eines »sowohl-als auch« zuzuordnen sein dürften. Wohl kann man - mit Otto - sagen, dass es für die Annahme

\footnotetext{
${ }^{16}$ H. Metzger, E. Laroche, A. Dupont-Sommer, M. Mayrhofer, Fouilles du Xanthos VI : La stèle trilingue du Létôon, Paris 1979.
} 
eines Ausgleichs zwischen den unterschiedlichen Konzeptionen im Pentateuch »nicht der These einer >Reichsautorisation< [bedarf] «, sondern dass dieser »ein notwendiger Schritt auf dem Weg zum Kanon ${ }^{17}{ }^{17}$ darstellt, doch ist der in der Tora beobachtbare Ausgleich sachlich unterschiedlich profilierter Konzeptionen nur eines ihrer besonderen Merkmale. Ebenso erklärungsbedürftig bleibt ihre Ausgrenzung als eigenständige literarische Größe, eben als »Tora«, aus dem Zusammenhang der Geschichtsbücher Gen-2Kön insgesamt.

\section{Lokale Normen und Zentralgewalt im Perserreich}

P. Frei definierte die persische Reichsautorisation »als ein Verfahren, durch das die von einer lokalen Instanz gesetzten Normen von einer Instanz der Zentrale nicht einfach gebilligt und akzeptiert, sondern übernommen und zur eigenen Norm gemacht werden. Die lokale Norm wird dadurch im Rahmen des gesamten staatlichen Verbandes, eben des Reiches, als Norm höheren Ranges für alle verbindlich gemacht und gesichert ${ }^{18}$. Diese Bestimmungen haben - nicht ohne Anhalt an den gewählten Formulierungen Anlass zu Missverständnissen gegeben, die so weit gehen, dass aufgrund dieser Missverständnisse die These insgesamt abgelehnt worden ist. Frei ging es im wesentlichen um den qualitativen Aspekt der Übernahme lokaler Normen durch die Zentralgewalt, die dadurch auf die Stufe der Reichsgesetzgebung erhoben werden. In der Diskussion wurde dieser qualitative Aspekt um ein nicht unwesentliches quantitatives Merkmal erweitert, das aber von Frei weder intendiert noch expliziert worden ist, dass nämlich die lokalen Normen zentral im Sinne eines »Reichsgesetzes« registriert und kodifiziert worden seien. J. Wiesehöfer scheint in seinem vielbeachteten Diskussionsbeitrag zur persischen Reichsautorisation (s. Anm. 13) die Aussagen von P. Frei in diesem Sinne aufgefasst zu haben: »[A]uf ein Reichszentralkataster, ein Reichszentralarchiv, das auch die speziellsten lokalen Regelungen notiert, gibt es keinen Hinweis.«Wiesehöfer gesteht so zwar durchaus zu, dass es im Perserreich Vorgänge der Ratifizierung lokaler Normen durch die Zentralgewalt gegeben habe. »Jedoch sehe ich, zumindest in den nichtalttestamentlichen Texten und in Esra, keinen Hinweis darauf gegeben, dass es so etwas wie ein >persisches Reichsgesetz $<$ gegeben hat, in das auch die lokalen Normen - nun als Reichsnormen - aufgenommen waren ${ }^{19}$.

\footnotetext{
${ }^{17}$ ZAW 1995, 375 Anm. 14.

${ }^{18}$ Frei, ZAR 1995, 3; vgl. 29.

19 44; ähnlich G. Ahn, »Toleranz« und Reglement. Die Signifikanz achaimenidischer Religionspolitik für den jüdisch-persischen Kulturkontakt, in: R.G. Kratz (Hg.), Religion und Religionskontakte im Zeitalter der Achämeniden, VWGTh 22, Gütersloh 2001, 191-209, 194 Anm. 18; G.N. Knoppers, An
} 
Das aber hat Frei nie behauptet. Es geht ihm um die staatsrechtliche Bedeutung der autorisierten lokalen Normen, nicht um deren zentrale Kodifizierung und Archivierung. Ein weiterer Einwand Wiesehöfers betrifft den Umstand, dass in den von Frei angeführten Beispielen der Großkönig selbst gar nicht involviert sei (44). Die Beobachtung ist zutreffend, doch sollte man diesen Befund nicht überbewerten, denn der Satrap repräsentiert außerhalb des Kernlandes, welches keinen Satrapen besitzt, selbstverständlich die Zentralregierung und nimmt deren Interessen in der jeweiligen Satrapie wahr ${ }^{20}$.

Weiter gehen die kritischen Anfragen an P. Frei, die U. Rüterswörden formuliert hat. Sie sind allerdings auch hypothetischerer Natur als die Einwände Wiesehöfers, da sie für die in der Demotischen Chronik ${ }^{21}$ sowie bei Diodor (I, 94f) überlieferte Nachricht, dass König Darios die ägyptischen Gesetze sammeln und aufzeichnen ließ, annehmen, dass es sich jeweils um hellenistische Rückprojektionen handelt: Das ägyptische Volksrecht der Ptolemäerzeit soll nach Rüterswörden durch seine perserzeitliche Anciennität legitimiert werden. Für die Rekonstruktion politischer Vorgänge oder Einrichtungen im Perserreich kämen diese Belege aber nicht in Frage. Rüterswörden kann zwar durchaus hellenistisches Kolorit der entsprechenden Quellen aufzeigen, doch lässt dies noch keinen zwingenden Schluss darauf zu, dass das von diesen Quellen Berichtete erst in der hellenistischen Zeit fingiert worden ist ${ }^{22}$.

Monographisch ausgebaut worden sind die Ansätze Rüterswördens durch seinen Schüler S. Grätz, der sie auf das Edikt des Artaxerxes in Esr 7,12-26 anwendet und dieses nun ebenfalls als hellenistische Fiktion interpretiert. Dieses Edikt ist für die Diskussion um die persische Reichsautorisation deshalb von Bedeutung, weil die unmittelbare Zusammenstellung von „Gesetz deines [sc. Esras] Gottes“ und „Gesetz des [sc. persischen] Königs“ in 7,26 in der Forschung oft so gedeutet worden ist, dass diese beiden Größen hier - im Sinne einer persischen Reichsautorisation von Esras Gesetz als identisch anzusehen seinen ${ }^{23}$. Formal entspreche dieses Edikt nach Grätz einer hellenistischen königlichen Schenkung. Allerdings mangelt es für eine sichere

\footnotetext{
Achaemenid Imperial Authorization of Torah in Yehud?, in: J.W. Watts (Hg.), Persia and Torah. The Theory of Imperial Authorization of the Pentateuch, SBL.SS 17, Atlanta 2001, 115-134, 134; L. Massmann, Persien und die Tora, ZAR 9 (2003), 238-250, 249.

${ }^{20}$ Zum Verhältnis der Satrapen zum Großkönig vgl. P. Briant, From Cyrus to Alexander. A History of the Persian Empire, Winona Lake 2000, 338-347. Rechtsangelegenheiten oblagen in der Regel dem Satrapen, der Großkönig konnte jedoch jederzeit eingreifen, wenn die lokale Bevölkerung an ihn appellierte (345).

${ }^{21}$ W. Spiegelberg, Die sogenannte Demotische Chronik des Pap. 215 der Bibliothèque Nationale zu Paris nebst den auf der Rückseite des Papyrus stehenden Texten, Demotische Studien 7, Leipzig 1914.

${ }^{22}$ Vgl. auch die Auseinandersetzung bei Frei, ZAR 1995, 5f Anm. 12.14.

${ }^{23}$ Vgl. etwa T. Willi, Juda - Jehud - Israel. Studien zum Selbstverständnis des Judentums in persischer Zeit, FAT 12, Tübingen 1995, 91-117; Knoppers, An Achaemenid Imperial Authorization; Blum, Esra.
} 
Entscheidung an Gattungsanalogien (139f; die Beispiele aus Esr 6,7-13; 8,9-24 und Ant. XII § 138-144 sind nicht zwingend), und die mit Achtergewicht versehenen Aussagen in Esr 7,25f verfolgen eine andere Zielrichtung als die einer Schenkung. Überdies wäre zu Grätz' Datierungsvorschlag grundsätzlich die Möglichkeit zu berücksichtigen, dass ein hellenistischer Text wie Esr 7 ältere, perserzeitliche Vorgänge einer »Reichsautorisation« rezipiert und diese nun im Sinne einer literarischen Stilisierung auf die Einführung der Tora in Juda überträgt ${ }^{24}$.

Weitere Einwände gegenüber Freis Theorie der Reichsautorisation hat Ska vorgetragen $^{25}$. Er weist auf die gattungsmäßige Heterogenität der von Frei herangezogenen Belege hin (164f), von denen nur Esr 7,25f; Neh 11,23 und die von Diodor bzw. der Demotischen Chronik bezeugten Gesetzessammlung unter Darius I. Fälle von nationaler Größenordnung behandeln. Der Befund, dass Vorgänge »persischer Reichsautorisation « in ganz unterschiedlichen Zusammenhängen dokumentiert sind, spricht allerdings prinzipiell nicht gegen die Theorie, sondern demonstriert vielmehr, dass diese Institution in verschiedener Weise und in unterschiedlicher Ausprägung Anwendung fand.

Nach Redford ${ }^{26}$ handelt es sich bei der Gesetzessammlung unter Darius I. nicht um »Gesetzgebung «, sondern dieser Eindruck entsteht aus der Rezeptionsperspektive Diodors, der Ägypten in »hellenisiertem《 Gewand, also auch mit einer eigenen Gesetzgebung erscheinen lassen will. Was die demotische Chronik beschreibe, habe - so Redford - weder eine Reichsautorisation noch eine Kodifikation ägyptischen Rechts im Blick, sondern vielmehr dessen Übesetzung ins Aramäische, in die lingua franca des Perserreichs, die es den neuen persischen Autoritäten erlaubte, die Reichtümer der ägyptischen Tempel $\mathrm{zu}$ kontrollieren und zu administrieren ${ }^{27}$.

Grabbe $^{28}$ erkennt anstelle einer streng gedachten »Reichsautorisation« der Tora durch Esra - bezüglich dessen Historizität er einige Zweifel hegt - eher »a permission« seitens der Perser, »to teach the >law in his hand $\ll{ }^{29}$; Knoppers $^{30}$ optiert ebenfalls für eine offenere Vorstellung des mit »Reichsautorisation« gemeinten Vorgangs. Er rechnet nicht mit einer stark zentralisierten und uniformierten persischen Politik der Autorisation lokaler Normen, sondern erkennt unterschiedliche Formen der Toleranz

\footnotetext{
${ }^{24}$ Dieser Einwand gilt auch gegenüber Pakkala, Ezra, 38.

${ }^{25}$ J.L. Ska, »Persian Imperial Authorization«: Some Question Marks, in: J.W. Watts (Hg.), Persia and Torah. The Theory of Imperial Authorization of the Pentateuch, SBL.SS 17, Atlanta 2001, 161-182.

${ }^{26}$ D.B. Redford, The So-Called »Codification« of Egyptian Law under Darius I, in: J.W. Watts (Hg.), Persia and Torah. The Theory of Imperial Authorization of the Pentateuch, SBL.SS 17, Atlanta 2001, $135-159$.

27158 .

${ }^{28}$ L.L. Grabbe, The Law of Moses in the Ezra Tradition: More Virtual Than Real?, in: J.W. Watts (Hg.), Persia and Torah. The Theory of Imperial Authorization of the Pentateuch, SBL.SS 17, Atlanta 2001, 91113.

${ }^{29}$ The Law of Moses, 112.

${ }^{30}$ S.o. Anm. 19.
} 
relativer lokaler Autonomie (134). Auch Blenkinsopp ${ }^{31}$ nimmt einen gewissen Abstand zu seiner bisherigen Befürwortung der These der $»$ Reichsautorisation ${ }^{32}$ ein, lehnt sie aber deswegen nicht ab: Die von Frei vorgeschlagene Fassung der »Reichsautorisation« sieht er nur in der Trilingue aus Xanthos belegt und hält sie nur für eines unter mehreren Mitteln persischer Politik, das in seiner Bedeutung möglicherweise nicht besonders wichtig war (46).

In eine etwas andere Richtung als die genannten Stimmen geht Fried ${ }^{33}$. Ihrer Auffassung nach bestand Esras Mission darin, persische Richter und Magistraten in der Provinz Juda zu installieren (Esr 7,25). Deren Basis in der Rechtssprechung bestand aber entsprechend den sonstigen Gepflogenheiten im Perserreich nicht in einem »Gesetzbuch«, sondern in der Orientierung an verschiedenen Edikten (dem »law of the king «) und an übergeordneten Vorstellungen von Recht und Gerechtigkeit (»the dāta of the god « [88]). Auf diesem Hintergrund versucht Fried, die Aktivitäten Nehemias entsprechend zu plausibiliseren.

Mit Grabbe, Knoppers und Blenkinsopp ist in der Tat eine uniform zugespitzte Form der Theorie der »persischen Reichsautorisation« abzulehnen, die sie mit der Vorstellung eines Zentralarchivs, einer zentralen Steuerung und einer zentralen Rolle des Großkönigs (anstelle eines Satrapen) verbindet. Die Erhebung lokaler Normen in den qualitativen Status von Reichsrecht bleibt jedoch ein im Alten Orient sonst unbekannter Vorgang, der auch ohne die Elemente einer theoretischen Universalgeltung dieser Lokalnormen im Reich und der zentralen Verschriftung und Archivierung auffällig genug und damit theoriebedürftig und theoriefähig bleibt.

\section{Persische Reichsautorisation und Abschluss der Tora}

Darf, ja muss man - in nicht künstlich zugespitzter - Weise nach wie vor mit (wie auch immer näher $\mathrm{zu}$ bestimmenden und $\mathrm{zu}$ differenzierenden) Vorgängen persischer Reichsautorisation lokaler Normen rechnen, so stellt sich nun in einem weiteren Schritt gleichwohl die Frage, inwieweit die Formierung der Tora mit dieser Institution in Zusammenhang $\mathrm{zu}$ bringen ist. Dabei sind bezüglich dieses Zusammenhangs verschiedene Möglichkeiten denkbar. Neben der einfachen, heute zumeist diskutierten These, ob die Bildung der Tora (oder ihrer Vorstufen) historisch mit dem Vorgang einer

\footnotetext{
${ }^{31}$ J. Blenkinsopp, Was the Pentateuch the Civic and Religious Constitution of the Jewish Ethnos in the Persian Period?, in: J.W. Watts (Hg.), Persia and Torah. The Theory of Imperial Authorization of the Pentateuch, SBL.SS 17, Atlanta 2001, 41-62.

${ }^{32}$ Vgl. o. Anm. 10.

${ }^{33}$ L. Fried, »You Shall Appoint Judges«: Ezra’s Mission and the Rescript of Artaxerxes, in: J.W. Watts (Hg.), Persia and Torah. The Theory of Imperial Authorization of the Pentateuch, SBL.SS 17, Atlanta 2001, 63-90.
} 
Reichsautorisation zu erklären sei oder nicht, ist auch zu fragen, ob das Alte Testament, namentlich in Esr 7, die rechtliche Inkraftsetzung der Tora nach dem ihm bekannten Vorbild einer persischen Reichsautorisation interpretiert. Die beiden Annahmen schließen sich zudem nicht aus, sondern sie sind auch kombinierbar.

Am entschiedensten urteilt Blum in dieser Frage, der die entscheidenden Schritte in der Komposition des Pentateuch in den Rahmen persischer Politik stellt: »(KD und ) KP [wurde] unter anderem auch unter der Perspektive der $>$ Reichsautorisation $<$ gestaltet ${ }^{34}$ : Die Zusammenstellung deuteronomistischer und priesterlicher Kompositionselemente im Pentateuch sei ohne äußeren Zwang gar nicht vorstellbar. Nun ist Blums Feststellung der »diskontinuierlichen Fügung «, mittels derer deuteronomistisches und priesterliches Material im Pentateuch textlich kombiniert worden sind, grundsätzlich zuzustimmen, denn die unterschiedliche Prägung dieser Texte ist so offensichtlich, dass sie selbst in der gegenwärtig diffusen Diskussionslage der Pentateuchforschung beinahe allgemein anerkannt ist. Doch das Argument ist nicht zwingend, was die Analogielosigkeit der pentateuchischen Verhältnisse betrifft. Auch in anderen Überlieferungsbereichen des Alten Testaments finden sich sachlich diametral entgegengesetzte Positionen, ohne dass für die Formierung des entsprechenden Buchs ein äußerer, politischer oder religiöser Zwang in Anspruch genommen würde. Ein deutliches Beispiel liefern etwa die golaund diasporaorientierten Aussagen im Jeremiabuch: In direkter Aufnahme revozieren diasporaorientierte Texte wie Jer 23,7f oder 29,*14 golaorientierte Gerichtsaussagen wie Jer 24,8-10 oder 29,16-19.

Der Zusammenschluss unterschiedlicher Konzeptionen innerhalb der Tora muss also nicht, er kann aber unter Zuhilfenahme der These einer persischen Reichsautorisation erklärt werden. Auch dagegen ist allerdings eine Reihe von Gegenargumenten vorgetragen worden. In seine Kritik an der These einer persischen Reichsautorisation als solcher hat Ska auch Überlegungen gegen deren Anwendung in bezug auf den Pentateuch eingeschlossen. So enthalte der Pentateuch vergleichsweise umfangreiches Erzählmaterial, das den Zwecken einer Reichsautorisation entgegenstehe, ja überdies quasiimperiale Ansprüche Israels vertrete (vgl. Gen 15,18), die kaum persischerseits gebilligt worden sein dürften. Außerdem sei das im Pentateuch enthaltene juridische Material aufgrund seiner Vielschichtigkeit, ja teilweisen Widersprüchlichkeit gar nicht justitiabel. Schließlich falle auf, dass der Pentateuch selbst keinen Hinweis auf seine in Frage stehende Autorisation durch die Perser enthalte ${ }^{35}$ und dass es auch keine

\footnotetext{
${ }^{34} \mathrm{Vgl}$. Komposition, 358; 360 mit Anm. 96; ders., Esra, 235-246.

${ }^{35}$ Von Schmitt, Suche, 264, nachgerade als Konstitutivum des Vorgangs persischer Reichsautorisation angesehen (»als von den persischen Behörden erlassene Gesetze formuliert«). Vgl. dagegen Blum, Esra, 251 Anm. 81.
} 
Hinweise auf eine zeitgenössische aramäische Übersetzung des Pentateuch gebe, wie dies angesichts der Bedeutung des Reichsaramäischen zu erwarten gewesen wäre.

Auf solche Bedenken hat Blum bereits mit einigen grundsätzlichen Überlegungen reagiert: Die neuzeitlicher Einschätzung in der Tat als Ineinander von Gesetz und Geschichte erscheinende Gestalt des Pentateuch muss nicht deckungsgleich sein mit der antiken Wahrnehmung. Vor allem aber gilt: »Der kritische Einwand schließlich, wie ein solch widersprüchliches >Kompromissdokument< als Rechtsgrundlage hätte fungieren sollen, übersieht den trivialen Tatbestand, $d a \beta$ die Tora seit der Antike eben diese Geltung hat $\ll^{36}$.

Der Hinweis auf Gen 15,18 ist bedenkenswert - gleicherweise ließen sich auch die Beschreibungen der kriegerischen Landnahme im Ostjordanland im Numeribuch nennen -, doch es ist die Frage, wie dieser Text historisch im Perserreich verstanden wurde. In theokratisch, perserloyaler Sicht konnte Gen 15,18 durchaus als in der Perserzeit erfüllt angesehen werden: Dadurch, dass sich die Siedlungsräume der Juden zu dieser Zeit von Mesopotamien bis Ägypten erstreckten, war diese Verheißung Realität geworden. Die Landnahmeberichte des Numeribuch als historisch-narrative Texte bereiteten in dieser Hinsicht ohnehin keine Schwierigkeiten.

Etwas mechanisch gedacht ist schließlich die aus der Elephantine-Korrespondenz gewonnene Argumentation Rüterswördens gegen die These einer Reichsautorisation der Tora: »Die Perser würden den Wiederaufbau einer Kultstätte gestatten, deren schiere Existenz allein schon gegen jenes Gesetz verstößt, das ja als persisches Reichsgesetz gelten soll. Schlimmer noch: Nicht der Satrap von Ägypten verordnet den Wiederaufbau, sondern die Autoritäten in Transeuphratene, wo nach Esr 7,25 die Thora gelten soll ${ }^{37}$. Gerade die »schiere Existenz « des Tempels in Elephantine bildete einen rechtsrelevanten Bestand: Im Falle seines Wiederaufbaus konfligierten aufgrund seines vormaligen Bestehens verschiedene Rechtsansprüche. Auf der einen Seite steht die Forderung der Kultzentralisation in der Tora (Dtn 12), auf der anderen das Gewohnheitsrecht der Wiederherstellung eines status quo (»Restoration of the status $q u o$ figured prominently in Persian thought and deed $\aleph^{38}$ ), das offenbar nicht von vornherein den Bestimmungen der Tora unterzuordnen war. Im übrigen gilt: »[G]erade

\footnotetext{
${ }^{36}$ Esra, 248.

${ }^{37}$ ZAR 1995, 60; vgl. auch Ska, »Persian Imperial Authorization«, 166 mit Anm. 16.

38 B. Porten, The Elephantine Papyri in English. Three Millennia of Cross-Cultural Continuity and Change, DMOA 22, Leiden u.a. 1996, 143 Anm. 79 (zur Erläuterung des Arguments in B19 [AP 30], 24f: »May a letter from you be sent to them about the Temple of YHW the God to (re)build it in Elephantine the fortress just as it had been build formerly«; vgl. auch B 21 [AP 32], 4f: »about the Altar-house of the God of Heaven which in Elephantine the fortress built was formerly before Cambyses « [Hervorhebungen K.S.]).
} 
an diesem Beispiel [wird] deutlich, daß/wie persische Behörden sich bei solchen Angelegenheiten inhaltlich (selbstverständlich) auf jüdische Urteilsbildung stützten. ${ }^{39}$

\section{Abschließende Bemerkungen}

Welche Schlüsse lassen sich aus diesen Überlegungen ziehen? Beurteilt man die These der persischen Reichsautorisation losgelöst von den Zuspitzungen durch ihre Kritiker, so sollte deutlich geworden sein, dass eine bloße Ablehnung eine zu einfache, ja in der Konsequenz unsachgemäße Option darstellt. Unterschiedlich akzentuierte Vorgänge der Autorisation lokaler Normen durch die persische Zentralgewalt sind durch Quellen hinreichend bezeugt. Allerdings implizieren solche Autorisationsvorgänge weder die Herstellung und Pflege eines zentralen Archivs der autorisierten Normen, die beständige Involvierung des persischen Großkönigs selbst in jeder einzelnen Autorisierungsfrage, noch eine notwendige Initiative persischerseits bei solchen Vorgängen. Das bedeutet nicht, dass damit von der These nicht mehr viel übrig bleibt zu betonen bleibt das im antiken Kontext innovative und analogielose Element der Ausstattung lokaler Normen mit der Autorität der persischen Zentralgewalt.

Wie die Formierung der Tora mit solchen Autorisationsvorgängen in Verbindung zu bringen ist, bleibt gegenwärtig eine offene Frage. Dass sie damit nichts zu tun gehabt hat, ist wenig wahrscheinlich. Dem steht der Artaxerxes-Firman Esr 7 entgegen - und zwar vollkommen unabhängig davon, ob man diesen Text nun als authentisch ansieht oder nicht, ihn perserzeitlich oder hellenistisch datiert. Denn Esr 7 zeigt, dass dem Verfasser dieses Textes Vorgänge der imperialen Autorisierung lokaler Normen bekannt waren - wie sie auch ohne weiteres in unterschiedlichen Spielarten aus weiteren Quellen belegbar sind - und dass er die Inkraftsetzung der Tora durch Esra seinen Lesern gegenüber entsprechend präsentierte. $\mathrm{Ob}$ das in historischem oder historisierend-fiktivem Sinn auszuwerten ist, muss vorerst dahingestellt bleiben. Jedenfalls bleibt weiter zu prüfen, ob der Abschluss der Tora (oder auch die Bildung maßgeblicher Vorstufen) tatsächlich als Produkt einer näher zu differenzierenden und präzisierenden Form persischer Reichsautorisation verstanden werden kann. Ein Zwang zu einer solchen Annahme aufgrund der »diskontinuierlichen Fügung« des in ihr enthaltenen Textguts besteht nicht, eine solche Annahme ist aber auch nicht ausgeschlossen. Ihre Schwierigkeiten sind in der jüngeren Diskussion oft betont worden, einige von ihnen sind allerdings kaum mehr als Phantome. Man sollte auf der

\footnotetext{
${ }^{39}$ Blum, Esra, 248f Anm. 70.
} 
anderen Seite nicht die Schwierigkeiten vergessen, die sich ergeben, wenn man auf sie verzichtet: Weshalb wurde der Pentateuch im wesentlichen in der Perserzeit abgeschlossen? Es sind dann bessere Vorschläge zu machen, weshalb der Pentateuch zur Tora avancieren konnte. Der Verweis darauf, dass er ein Produkt jüdischer Schriftgelehrsamkeit sei, reicht dabei nicht aus, denn das gilt vom gesamten Alten Testament. 